\title{
AN ACTIVE-SET MIXED FINITE ELEMENT SOLVER FOR A TRANSIENT HYDRODYNAMIC LUBRICATION PROBLEM IN THE PRESENCE OF CAVITATION.
}

\author{
MOULAY HICHAM TBER
}

\begin{abstract}
In this paper we study a moving free boundary problem related to the the cavitation modeling in lubricated devices. More precisely, a characteristics method combined with a weak formulation in a mixed form is introduced for the Elrod-Adams model. The formulation is suitable for the use of mixed finite element methods in the numerical approximation. It is proved that the time-discrete problem and its finite element discretization has a unique solution. Further an efficient primal-dual active-set strategy is proposed to solve the resulting algebraic system. The performance of the overall algorithm is illustrated by numerical examples.
\end{abstract}

\section{INTRODUCTION}

For a long time, Reynolds equation has been used to describe the behavior of a viscous flow inside the lubricated devices. Nevertheless, Reynolds modeling approach does not take into account the rupture of the continuous lubricant film due to the formation of air bubbles. This phenomenon, called cavitation, is not only important because its onset and extent determine the performance of the lubricated device but also because vapor cavitation collapse (implosion) can cause severe surface material damage [18]. Thus, various models have been used in order to make the Reynolds equation valid in the cavitation area. The Elrod-Adams modeling approach [11], here adopted, is one of the most realistic approaches taking account of the cavitation phenomena. In this model, the cavitation region is considered as a fluid-air mixture and an additional unknown representing the saturation of the fluid in the mixture is introduced. The study of this model has given place to many works covering some fundamental and applied works (see $[6,13]$ and the references within).

Our motivation in this paper is to design an efficient numerical solution algorithm based on an appropriate time-space discretization for the Elrod-Adams cavitation

Date: October 3, 2018.

2000 Mathematics Subject Classification. 76T10, 35L87, 65M25, 65N30, 49M15.

Key words and phrases. Cavitation, Elrod-Adams model, characteristics, mixed formulation, active set solver. 
model. By considering flow along the characteristics we derive a mixed variational formulation of the problem. We rewrite the semi-discrete governing equations in the form of system of first order equations in which the flux is treated as an independent variable. We mention here that a such approach has been already used for advection-dominated transport problems [2]. Here it is shown that the semi-discrete problem has a unique solution by using Shauder's fixed point theorem and a regularization technique. Moreover, the weak solution is approximated by a mixed finite element (MFE) method. A primal-dual active set strategy which is equivalent to a semi-smooth Newton algorithm is used to solve the complementarity-saddle point problem arising from the characteristics-MFE discretization.

The rest of this paper is organized as follows: In section 2 the mathematical formulation of the problem is presented. The set of equations defines a nonlinear advection diffusion free boundary problem where the free boundary separates the lubricated and cavitated regions. In section 3, we approximate the hyperbolic part of the equation along the characteristics. In section 4 , we derive a weak formulation of the semi-discrete problem in a mixed form and we prove the existence of one weak solution. In the same section we approximate the weak solution by a mixed finite element method. In section 5 , we design a solution algorithm for the resulting nonlinear algebraic equation. Numerical experiments are reported in section 6 .

\section{THE MATHEMATICAL MODEL}

Studying the cavitation phenomenon in lubricated devices gives place to a mathematical formulation in a domain $\Omega \subset \mathbb{R}^{n}$ with $n=1,2$ of regular boundary $\Gamma=\Gamma_{i} \cup \Gamma_{e}$ with $\Gamma_{i} \cap \Gamma_{e}=\emptyset$. According to Elrod-Adams model, the unknowns of the problem are the pressure $p(x, t)$ and the relative content $\theta(x, t)$ of oil film with $x \in \Omega$ and $t_{0} \leq t \leq T$. When the lubrication takes place by an incompressible fluid of viscosity $\mu$, the pressure satisfies the Reynolds equation:

$$
\frac{\partial h}{\partial t}-\nabla\left(\frac{h^{3}}{12 \mu} \nabla p-\frac{h}{2} U \boldsymbol{e}_{1}\right)=0, \quad \theta=1, \quad \text { in } \Omega^{+}=\left\{x \in \Omega \mid p(x)>p_{c}\right\}
$$

and $\theta$ satisfies the conservation law

$$
\frac{\partial(h \theta)}{\partial t}+\nabla\left(\frac{h}{2} \theta U \boldsymbol{e}_{1}\right)=0, \quad 0 \leq \theta \leq 1, \quad \text { in } \quad \Omega^{0}=\left\{x \in \Omega \mid p(x)=p_{c}\right\},
$$

where $p_{c} \in \mathbb{R}$ is the cavitation pressure, $\boldsymbol{e}_{1}$ is the unit vector in the $x_{1}$-direction and $U(x, t)$ is the relative sliding speed of the contact surfaces with $U \boldsymbol{e}_{1}$ being a divergence-free vector field. The function $h=h(x, t)$ that represents the film 
thickness satisfies

$$
\left.\exists h_{0}, h_{1} \in \mathbb{R}_{+}^{*}, \quad \forall(x, t) \in \Omega \times\right] t_{0}, T\left[, \quad h_{0} \leq h(x, t) \leq h_{1} .\right.
$$

On the free boundary $\Sigma=\partial \Omega^{+} \cap \Omega$, we have the conservation condition of the flux

$$
\left(h^{0} \theta^{0}-h^{+}\right) \frac{U}{2} \boldsymbol{e}_{1} \cdot \mathbf{n}+\frac{\left(h^{+}\right)^{3}}{12 \mu}\left(\frac{\partial p}{\partial \mathbf{n}}\right)^{+}=\left(h^{0} \theta^{0}-h^{+}\right) \mathbf{V}_{\Sigma} \cdot \mathbf{n},
$$

where $\mathbf{n}$ is the unit vector normal on $\Sigma$ oriented outwards from $\Omega^{+} . \mathrm{V}_{\Sigma}$ is the velocity of $\Sigma$. The superscripts 0 and + refers to the limit values of the corresponding quantities as $\Sigma$ is approached from the cavitated and full film sides respectively.

The Reynolds equation (2.1) and the conservation law (2.2) lead to the following equation valid in both cavitation and lubricated region:

$$
\frac{\partial \theta h}{\partial t}+\nabla\left(\frac{h}{2} \theta U \boldsymbol{e}_{1}\right)-\nabla\left(\frac{h^{3}}{12 \mu} \nabla p\right)=0
$$

The pressure $p$ and the concentration $\theta$ are related by

$$
p \geq 0 \quad 0 \leq \theta \leq 1 \quad p(1-\theta)=0 .
$$

Here and in the following, it is assumed without a loss of generality that the cavtitaion pressure $p_{c}=0$. Thus the strong formulation of the problem is given by the following set of equations:

$$
\left\{\begin{array}{c}
\frac{\partial(h \theta)}{\partial t}+\frac{U}{2} \boldsymbol{e}_{1} \cdot \nabla(h \theta)-\nabla\left(\frac{h^{3}}{12 \mu} \nabla p\right)=0 \\
p \geq 0, \quad p(1-\theta)=0, \quad 0 \leq \theta \leq 1, \\
\left.p\right|_{\Gamma_{i}}=p_{i},\left.\quad p\right|_{\Gamma_{e}}=p_{e}
\end{array}\right.
$$

where $p_{i}=p_{i}(t)$ and $p_{e}=p_{e}(t)$ are given supply pressures.

We supplement the above system by an appropriate initial condition $\theta\left(t=t_{0}\right)=\theta_{0}$ with $0 \leq \theta_{0}(x) \leq 1$ for $x \in \Omega$.

\section{TIME DISCRETIZATION}

Due to the hyperbolic character of the governing equation in cavitated areas, the numerical solutions of (2.4) may exhibit undesired oscillations. Following [5], one possible solution to deal with this issue consists in writing $\frac{\partial(h \theta)}{\partial t}+\frac{U}{2} \boldsymbol{e}_{1} \cdot \nabla(h \theta)$ as $\frac{D(h \theta)}{D t}$ the material derivative of $h \theta$ in the direction of $\frac{U}{2} \boldsymbol{e}_{1}$. Then we can rewrite the first equation in (2.4) as

$$
\frac{D(h \theta)}{D t}-\nabla\left(\frac{h^{3}}{12 \mu} \nabla p\right)=0 .
$$


The corresponding characteristic curves are defined by

$$
\left\{\begin{array}{c}
\frac{d X(x, t ; s)}{d s}=\frac{U(x, s)}{2} \boldsymbol{e}_{1}, \\
X(x, t ; t)=x,
\end{array}\right.
$$

with $X(x, t ; s)$ being the position of a particle at time $s$, which was or will be at $x$ at time $t$.

Now for a given uniform time step size $\tau>0$, we can get an approximate value of $X$ at $t^{\text {old }}=t-\tau$ by

$$
X\left(x, t ; t^{o l d}\right)=x-\tau \frac{U(x, t)}{2} \boldsymbol{e}_{1} .
$$

Using a fully-implicit scheme, we get the semi-discrete form of (2.4)

$$
\left\{\begin{array}{c}
h \theta-\nabla\left(\frac{\tau h^{3}}{12 \mu} \nabla p\right)=h^{\text {old }} \theta^{\text {old }} \\
p \geq 0, \quad p(1-\theta)=0, \quad 0 \leq \theta \leq 1, \\
\left.p\right|_{\Gamma_{i}}=p_{i},\left.\quad p\right|_{\Gamma_{e}}=p_{e}, \\
\theta(t=0)=\theta_{0},
\end{array}\right.
$$

where $\theta^{\text {old }}(x, t)=\theta\left(X\left(x, t ; t^{\text {old }}\right), t^{\text {old }}\right)$ and $h^{\text {old }}(x, t)=h\left(X\left(x, t ; t^{\text {old }}\right), t^{\text {old }}\right)$. This time approximation will be combined with a spatial discretization by a mixed finite element method.

\section{MIXED FORMULATION}

In this section, we introduce a weak formulation for the semi-discrete problem (3.2) in a mixed form. First we introduce the Hilbert space

$$
H(\text { div })=\left\{\mathbf{v} \in L^{2}(\Omega)^{n}, \nabla \cdot \mathbf{v} \in L^{2}(\Omega)\right\}
$$

and the associated norm

$$
\|\mathbf{v}\|_{H(d i v)}=\sqrt{\|\mathbf{v}\|_{0}^{2}+\|\nabla \cdot \mathbf{v}\|_{0}^{2}}
$$

where $\|\cdot\|_{0}$ denotes the standard $L^{2}$ norm for either vector-valued or real-valued functions as appropriate.

Next we define the velocity $\mathbf{u}$ by the following relation:

$$
\frac{12 \mu}{\tau h^{3}} \mathbf{u}+\nabla p=0 .
$$

It follows from (3.2) that

$$
h \theta+\nabla \cdot \mathbf{u}=h^{\text {old }} \theta^{\text {old }} .
$$

By testing (4.1) against any vector valued $\mathbf{v} \in H($ div), we obtain

$$
\left(\frac{12 \mu}{\tau h^{3}} \mathbf{u}, \mathbf{v}\right)+(\nabla p, \mathbf{v})=0 .
$$


Using Green's formula, one arrives at

$$
\left(\frac{12 \mu}{\tau h^{3}} \mathbf{u}, \mathbf{v}\right)-(\nabla \cdot \mathbf{v}, p)=-<p_{\Gamma}, \mathbf{v} \cdot n>_{\Gamma_{D}},
$$

where $(\cdot, \cdot)$ and $<\cdot, \cdot>_{\Gamma}$ indicates the inner product on $L^{2}(\Omega)$ and $L^{2}(\Gamma)$ respectively and $p_{\Gamma}$ denotes the trace of the pressure $p$ on the boundary $\Gamma$ :

$$
p_{\Gamma}=\left\{\begin{array}{lll}
p_{i} & \text { on } & \Gamma_{i}, \\
p_{e} & \text { on } & \Gamma_{e} .
\end{array}\right.
$$

Finally, by testing (4.2) against any $q \in L^{2}(\Omega)$ we obtain

$$
(h \theta, q)+(\nabla \cdot \mathbf{u}, q)=\left(h^{\text {old }} \theta^{\text {old }}, q\right) .
$$

Hence, the weak formulation of the problem consists in finding $p \in L^{2}(\Omega), \theta \in$ $L^{\infty}(\Omega)$ and $\mathbf{u} \in H($ div $)$ such that

$$
\left\{\begin{array}{l}
\left(\frac{12 \mu}{\tau h^{3}} \mathbf{u}, \mathbf{v}\right)-(\nabla \cdot \mathbf{v}, p)=-<p_{\Gamma}, \mathbf{v} \cdot n>_{\Gamma} \quad \forall \mathbf{v} \in H(\text { div }), \\
(\nabla \cdot \mathbf{u}, q)+(h \theta, q)=\left(h^{\text {old }} \theta^{\text {old }}, q\right) \quad \forall q \in L^{2}(\Omega), \\
p \geq 0, \quad p(1-\theta)=0, \quad 0 \leq \theta \leq 1, \quad \text { a.e. in } \Omega .
\end{array}\right.
$$

To show that the weak formulation has a solution, let $H_{\varepsilon}$ be a regularization of the Heaviside operator $H$ given by

$$
H_{\varepsilon}(x)= \begin{cases}1 & \text { if } x \geq \varepsilon \\ x / \varepsilon & \text { if } 0 \leq x \leq \varepsilon \\ 0 & \text { if } x \leq 0\end{cases}
$$

Correspondingly, we consider the following regularized problem

$$
\left\{\begin{aligned}
\text { Find } p_{\varepsilon} \in L^{2}(\Omega) \text { and } \mathbf{u}_{\varepsilon} \in H(\text { div }) \text { such that } & \\
p_{\varepsilon} & \geq 0 \quad \text { a.e. in } \Omega, \\
\left(\frac{12 \mu}{\tau h^{3}} \mathbf{u}_{\varepsilon}, \mathbf{v}\right)-\left(\nabla \cdot \mathbf{v}, p_{\varepsilon}\right) & =-<p_{\Gamma}, \mathbf{v} \cdot n>_{\Gamma} \quad \forall \mathbf{v} \in H(\text { div }), \\
\left(\nabla \cdot \mathbf{u}_{\varepsilon}, q\right)+\left(h H_{\varepsilon}\left(p_{\varepsilon}\right), q\right) & =\left(h^{\text {old }} \theta^{\text {old }}, q\right) \quad \forall q \in L^{2}(\Omega) .
\end{aligned}\right.
$$

Lemma 1. The regularized problem (4.7) has a unique solution $\left(\mathbf{u}_{\varepsilon}, p_{\varepsilon}\right)$. Moreover there exists a constant $C$ not depending on $\varepsilon$ such that

$$
\left\|\mathbf{u}_{\varepsilon}\right\|_{H(d i v)}+\left\|p_{\varepsilon}\right\|_{0} \leq C .
$$

Proof. Consider the mapping $T$ which, for any $p_{\varepsilon} \in L^{2}(\Omega)$, associates $\tilde{p}_{\varepsilon}=T\left(p_{\varepsilon}\right)$ the solution of the following problem:

$$
\begin{aligned}
\left(\frac{12 \mu}{\tau h^{3}} \widetilde{\mathbf{u}}_{\varepsilon}, \mathbf{v}\right)-\left(\nabla \cdot \mathbf{v}, \tilde{p}_{\varepsilon}\right) & =-<p_{\Gamma}, \mathbf{v} \cdot n>_{\Gamma} \quad \forall \mathbf{v} \in H(\text { div }), \\
\left(\nabla \cdot \widetilde{\mathbf{u}}_{\varepsilon}, q\right)+\left(h H_{\varepsilon}\left(p_{\varepsilon}\right), q\right) & =\left(h^{\text {old }} \theta^{\text {old }}, q\right) \quad \forall q \in L^{2}(\Omega),
\end{aligned}
$$


The problem (4.9) has a unique solution by the inf-sup condition of Brezzi and Babuska $[4,8]$. Moreover, there exists a constant $C=C\left(\Omega, p_{\Gamma}, h_{0}, h_{1}\right)$ such that

$$
\left\|\widetilde{\mathbf{u}}_{\varepsilon}\right\|_{H(d i v)}+\left\|\tilde{p}_{\varepsilon}\right\|_{0} \leq C\left(\Omega, p_{\Gamma}, h_{0}, h_{1}, \mu, \tau\right) .
$$

Here, we have used the fact that $\left(H_{\varepsilon}\left(\widetilde{\mathbf{u}}_{\varepsilon}\right)\right)_{\varepsilon}>0$ is bounded in $L^{\infty}(\Omega)$ independently of $\varepsilon$.

Notice that the first equation in (4.9) implies that

$$
\nabla \tilde{p}_{\varepsilon}=-\frac{12 \mu}{\tau h^{3}} \widetilde{\mathbf{u}}_{\varepsilon}
$$

is a function in $L^{2}(\Omega)^{2}$. This together with the estimate (4.10) shows that $T\left(p_{\varepsilon}\right)=$ $\tilde{p}_{\varepsilon} \in H^{1}(\Omega)$ and

$$
\left\|T\left(p_{\varepsilon}\right)\right\|_{1} \leq C\left(\Omega, p_{\Gamma}, h_{0}, h_{1}, \mu, \tau\right) .
$$

The mapping $T$ is then bounded from $L^{2}(\Omega)$ to $H^{1}(\Omega)$. From the compact embedding of $H^{1}(\Omega)$ into $L^{2}(\Omega)$, it follows that $T$ is completely continuous from $H^{1}(\Omega)$ to $L^{2}(\Omega)$. Moreover the estimate (4.11) shows that $T\left(B_{C}\right) \subset B_{C}$ with $B_{C}$ being the $H^{1}(\Omega)$-ball of radius $C\left(\Omega, p_{\Gamma}, h_{0}, h_{1}, \mu, \tau\right)$. Schauder fixed point theorem yields the existence of a function $p_{\varepsilon}$ such that $T\left(p_{\varepsilon}\right)=p_{\varepsilon}$. The fixed point $p_{\varepsilon}$ together with the corresponding $\mathbf{u}_{\varepsilon}$, forms a solution of the two equations in (4.7) satisfying (4.8) with $C=C\left(\Omega, p_{\Gamma}, h_{0}, h_{1}, \mu, \tau\right)$.

Next, we claim that $p_{\varepsilon} \geq 0$ a.e. in $\Omega$. Actually, $p_{\varepsilon}$ is also a solution of the following problem:

$$
\left\{\begin{aligned}
\text { Find } p_{\varepsilon} \in H^{1}(\Omega) \text { such that } & \\
p_{\varepsilon} & =p_{\Gamma} \quad \text { on } \Gamma, \\
h H_{\varepsilon}\left(p_{\varepsilon}\right)-\operatorname{div}\left(\frac{\tau h^{3}}{12 \mu} \nabla p_{\varepsilon}\right) & =h^{\text {old }} \theta^{\text {old }} \quad \forall q \in H_{0}^{1}(\Omega) .
\end{aligned}\right.
$$

Let $p_{\varepsilon}^{-}=\min \left(0, p_{\varepsilon}\right)$. It is clear that $p_{\varepsilon}^{-} \in H_{0}^{1}(\Omega)$. By choosing $q=p_{\varepsilon}^{-}$in (4.12) we arrive at

$$
\left(\frac{\tau h^{3}}{12 \mu} \nabla p_{\varepsilon}, \nabla p_{\varepsilon}^{-}\right)+\left(h H_{\varepsilon}\left(p_{\varepsilon}\right), p_{\varepsilon}^{-}\right)=\left(h^{o l d} \theta^{o l d}, p_{\varepsilon}^{-}\right) .
$$

Thus, using the fact that $H_{\varepsilon}(x)=0$ for $x \leq 0$ and $h^{\text {old }} \theta^{\text {old }} \geq 0$ we obtain

$$
\left(h^{3} \nabla p_{\varepsilon}^{-}, \nabla p_{\varepsilon}^{-}\right) \leq 0,
$$

which leads to $p_{\varepsilon}^{-}=0$ a.e. in $\Omega$ and then $p_{\varepsilon} \geq 0$ a.e. in $\Omega$. Consequently, the solution $\left(\mathbf{u}_{\varepsilon}, p_{\varepsilon}\right)$ is a solution of (4.7).

Theorem 2. There exists a unique triple $(\mathbf{u}, p, \theta) \in H($ div $) \times L^{2}(\Omega) \times L^{\infty}(\Omega)$ which is a solution of the weak formulation (4.6). 
Proof. Proof. For any $\varepsilon>0$, let $\left(\mathbf{u}_{\varepsilon}, p_{\varepsilon}\right)$ be the solution of (4.7). From (4.8) we can find a subsequence, also denoted $\left(\mathbf{u}_{\varepsilon}, p_{\varepsilon}\right)$, such that

$$
\begin{aligned}
p_{\varepsilon} \rightarrow p & \text { in } H^{1}(\Omega), \\
p_{\varepsilon} \longrightarrow p & \text { in } L^{2}(\Omega), \\
\mathbf{u}_{\varepsilon} \rightarrow \mathbf{u} & \text { in } H(d i v), \\
H_{\varepsilon}\left(p_{\varepsilon}\right) \rightarrow \theta & \text { in } L^{2}(\Omega), \\
H_{\varepsilon}\left(p_{\varepsilon}\right) \stackrel{*}{\rightarrow} \theta & \text { in } L^{\infty}(\Omega) .
\end{aligned}
$$

By passing to the limit in (4.7), we deduce that

$$
\left\{\begin{aligned}
p & \geq 0 \quad \text { a.e. in } \Omega \\
\left(\frac{12 \mu}{\tau h^{3}} \mathbf{u}, \mathbf{v}\right)-(\nabla \cdot \mathbf{v}, p) & =-<p_{\Gamma}, \mathbf{v} \cdot n>_{\Gamma} \\
(\nabla \cdot \mathbf{u}, q)+(h \theta, q) & =\left(h^{\text {old }} \theta^{\text {old }}, q\right) .
\end{aligned}\right.
$$

To complete the proof of existence of a solution for the initial problem (4.6), it remains to prove that $0 \leq \theta \leq 1$ and $p(1-\theta)=0$ a.e. in $\Omega$.

First, since

$$
H_{\varepsilon}\left(p_{\varepsilon}\right) \stackrel{*}{\rightarrow} \theta \text { in } L^{\infty}(\Omega)
$$

we have

$$
1-H_{\varepsilon}\left(p_{\varepsilon}\right) \stackrel{*}{\rightarrow} 1-\theta \text { in } L^{\infty}(\Omega) .
$$

Hence

$$
\|\theta\|_{\infty} \leq \liminf H_{\varepsilon}\left(p_{\varepsilon}\right) \leq 1 \text { and }\|1-\theta\|_{\infty} \leq \liminf \left(1-H_{\varepsilon}\left(p_{\varepsilon}\right)\right) \leq 1,
$$

i.e.

$$
0 \leq \theta \leq 1
$$

Furthermore, one has

$$
p_{\varepsilon}\left(1-H_{\varepsilon}\left(p_{\varepsilon}\right)\right) \rightarrow p(1-\theta) \text { in } L^{2}(\Omega) .
$$

Indeed, for $\phi$ in $L^{2}(\Omega)$ we have

$$
\begin{aligned}
\left(p_{\varepsilon}\left(1-H_{\varepsilon}\left(p_{\varepsilon}\right)\right)-p(1-\theta), \phi\right) & =\left(\left(p_{\varepsilon}-p\right)\left(1-H_{\varepsilon}\left(p_{\varepsilon}\right)\right), \phi\right)+\left(p\left(\theta-H_{\varepsilon}\left(p_{\varepsilon}\right)\right), \phi\right) \\
& \leq\left\|p_{\varepsilon}-p\right\|_{0}\|\phi\|_{0}+\left|\left(\left(\theta-H_{\varepsilon}\left(p_{\varepsilon}\right)\right), p \phi\right)\right| .
\end{aligned}
$$

Since $p \phi \in L^{1}(\Omega)$, we get (4.14) from the $L^{2}(\Omega)$ strong convergence of $p_{\varepsilon}$ to $p$ and the $L^{\infty}(\Omega)$ weak-* convergence of $H_{\varepsilon}\left(p_{\varepsilon}\right)$ to $\theta$.

On the other hand, from $H_{\varepsilon}$ expression, we have

$$
\left(p_{\varepsilon}\left(1-H_{\varepsilon}\left(p_{\varepsilon}\right)\right), \phi\right) \leq \varepsilon\|\phi\|_{0},
$$

whence

$$
p_{\varepsilon}\left(1-H_{\varepsilon}\left(p_{\varepsilon}\right)\right) \rightarrow 0 \text { in } L^{2}(\Omega) .
$$


Consequently, from the uniqueness of the limit in $L^{2}(\Omega)$, we deduce that $p(1-\theta)=$ 0 a.e. in $\Omega$.

Finally, notice that the complementarity system

$$
\left\{\begin{array}{c}
\nabla \cdot \mathbf{u}+h \theta=h^{\text {old }} \theta^{\text {old }} \quad \text { in } L^{2}(\Omega), \\
p \geq 0, \quad p(1-\theta)=0, \quad 1-\theta \geq 0, \quad \text { a.e. in } \Omega,
\end{array}\right.
$$

and the variational inequality

$$
\left\{\begin{array}{l}
p \in L_{+}^{2}(\Omega):=\left\{q \in L^{2}(\Omega): \quad q \geq 0\right\}, \\
(\nabla \cdot \mathbf{u}, p-q) \leq\left(h^{\text {old }} \theta^{\text {old }}-h, p-q\right), \quad \forall q \in L_{+}^{2}(\Omega)
\end{array}\right.
$$

are equivalent. Therefore, for any $(\mathbf{u}, p, \theta)$ satisfying $(4.6),(\mathbf{u}, p)$ is a solution of the the following mixed variational inequality

$$
\left\{\begin{array}{l}
p \in L_{+}^{2}(\Omega):=\left\{q \in L^{2}(\Omega): \quad q \geq 0\right\}, \\
\left(\frac{12 \mu}{\tau h^{3}} \mathbf{u}, \mathbf{v}\right)-(\nabla \cdot \mathbf{v}, p)=-<p_{\Gamma}, \mathbf{v} \cdot n>_{\Gamma}, \quad \forall \mathbf{v} \in H(\text { div }), \\
(\nabla \cdot \mathbf{u}, p-q) \leq\left(h^{\text {old }} \theta^{\text {old }}-h, p-q\right), \quad \forall q \in L_{+}^{2}(\Omega)
\end{array}\right.
$$

which has a at most one solution by virtue of [9, Theorem 2.1]. The uniqueness of $\theta$ follows from (4.16) and (2.3).

The weak formulation (4.6) allows us to approximate the underlying lubrication problem by using mixed finite element methods. To this end, let $\mathcal{T}_{h}$ be a finite element partition of $\Omega$ into triangles. We consider a stable pair $\left(\mathcal{V}_{h}, Q_{h}\right)$ of spaces on $\mathcal{T}_{h}$ such that $p_{h} \in Q_{h} \subset L^{2}(\Omega)$ and $\mathbf{u}_{h} \in \mathcal{V}_{h} \subset H($ div $)$.

Our mixed finite element problem consists in finding a triple $\left(p_{h}, \mathbf{u}_{h}, \theta_{h}\right)$ in $Q_{h} \times$ $\mathcal{V}_{h} \times Q_{h}$ satisfying

$$
\left\{\begin{array}{l}
\left(\frac{12 \mu}{\tau h^{3}} \mathbf{u}_{h}, \mathbf{v}_{h}\right)-\left(\nabla \cdot \mathbf{v}_{h}, p_{h}\right)=-<p_{\Gamma}, \mathbf{v}_{h} \cdot n>_{\Gamma} \quad \forall \mathbf{v}_{h} \in \mathcal{V}_{h}, \\
\left(\nabla \cdot \mathbf{u}_{h}, q_{h}\right)+\left(h \theta_{h}, q_{h}\right)=\left(h^{\text {old }} \theta_{h}^{\text {old }}, q_{h}\right) \quad \forall q_{h} \in Q_{h}, \\
p_{h} \geq 0, \quad p_{h}\left(1-\theta_{h}\right)=0, \quad 0 \leq \theta_{h} \leq 1, \quad \text { a.e. in } \Omega .
\end{array}\right.
$$

Theorem 3. The discrete problem (4.19) has a unique solution.

Proof. By considering the following discrete regularized problem

$$
\left\{\begin{aligned}
\text { Find } p_{h, \varepsilon} \in Q_{h} \text { and } \mathbf{u}_{\mathrm{h}, \varepsilon} \in \mathcal{V}_{h} \text { such that } & \\
p_{h, \varepsilon} & \geq 0 \quad \text { a.e. in } \Omega, \\
\left(\frac{12 \mu}{\tau h^{3}} \mathbf{u}_{\mathbf{h}, \varepsilon}, \mathbf{v}_{h}\right)+\left(\nabla \cdot \mathbf{v}_{h}, p_{h, \varepsilon}\right) & =-<p_{\Gamma}, \mathbf{v}_{h} \cdot n>_{\Gamma} \quad \forall \mathbf{v}_{h} \in \mathcal{V}_{h}, \\
\left(\nabla \cdot \mathbf{u}_{\mathrm{h}, \varepsilon}, q_{h}\right)+\left(h H_{\varepsilon}\left(p_{h, \varepsilon}\right), \mathbf{v}_{h}\right) & =\left(h^{\text {old }} \theta^{\text {old }}, q_{h}\right) \quad \forall q_{h} \in Q_{h},
\end{aligned}\right.
$$

this theorem can be proven similarly to the previous one. In particular it can be shown that the solution of (4.19) is given by the unique solution of the following 
discrete mixed variational inequality

$$
\left\{\begin{array}{l}
p_{h} \in Q_{h}^{+}:=\left\{q_{h} \in Q_{h}: \quad q_{h} \geq 0\right\}, \\
\left(\frac{12 \mu}{\tau h^{3}} \mathbf{u}_{h}, \mathbf{v}_{h}\right)-\left(\nabla \cdot \mathbf{v}_{h}, p_{h}\right)=-<p_{\Gamma}, \mathbf{v}_{h} \cdot n>_{\Gamma} \quad \forall \mathbf{v}_{h} \in \mathcal{V}_{h}, \\
\left(\nabla \cdot \mathbf{u}_{h}, p_{h}-q_{h}\right)+\left(h \theta_{h}, p_{h}-q_{h}\right) \leq\left(h^{\text {old }} \theta^{\text {old }}, p_{h}-q_{h}\right) \quad \forall q_{h} \in Q_{h}^{+} .
\end{array}\right.
$$

\section{Active set strategy}

The solution algorithm we are proposing in this section relies on the complementarity formulation of (4.21). More precisely we consider the following system

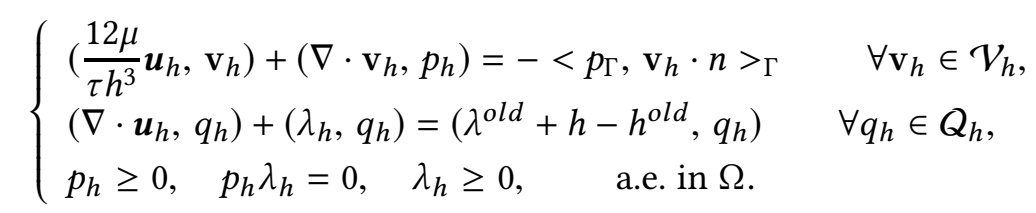

For sake of convenience we have made the following change of variables

$$
\lambda_{h}=h\left(1-\theta_{h}\right) \quad \boldsymbol{u}_{h}=-\mathbf{u}_{h} \quad \lambda^{\text {old }}=h^{\text {old }}\left(1-\theta^{\text {old }}\right) .
$$

The matrix form of (4.21) reads

$$
\left\{\begin{array}{l}
M U+B P=F_{v} \\
B^{T} U+D \Lambda=F_{q} \\
P \geq 0, \quad P^{T} \Lambda=0, \quad \Lambda \geq 0,
\end{array}\right.
$$

where $U, P$ and $\Lambda$ are the algebraic representation of $\boldsymbol{u}_{h}, p_{h}$ and $\lambda_{h}$ with respect to the basis of $\mathcal{V}_{h}$ and $Q_{h}$. The matrix blocks and the right side vectors are given by

$$
\begin{gathered}
M=\left[\left(\frac{12 \mu}{\tau h^{3}} \mathbf{v}_{\mathbf{i}}, \mathbf{v}_{\mathbf{j}}\right)\right]_{i, j=1}^{n_{u}} \quad B=\left[\left(\nabla \cdot \mathbf{v}_{\mathbf{j}}, q_{i}\right)\right]_{i, j=1}^{n_{p}, n_{u}} \quad D=\left[\left(q_{i}, q_{j}\right)\right]_{i, j=1}^{n_{p}} \\
F_{v}=\left[<p_{\Gamma}, \mathbf{v}_{\mathbf{i}} \cdot \boldsymbol{n}>_{\Gamma}\right]_{i=1}^{n_{u}}, \quad F_{q}=\left[\left(\lambda^{\text {old }}+\left(h-h^{\text {old }}\right), q_{i}\right)\right]_{i=1}^{n_{p}} .
\end{gathered}
$$

By $\left(\mathbf{v}_{\mathbf{i}}\right)_{i=1}^{n_{u}}$ and $\left(q_{i}\right)_{i=1}^{n_{p}}$ we denote the basis functions of the spaces $\mathcal{V}_{h}$ and $Q_{h}$ respectively.

Now for a fixed parameter $c>0$, we introduce the active and inactive sets

$$
A:=\left\{i \in\left\{1, \ldots, n_{p}\right\} \mid \Lambda_{i}-c P_{i}>0\right\}, \quad I:=\left\{i \in\left\{1, \ldots, n_{p}\right\} \mid \Lambda_{i}-c P_{i} \leq 0\right\} .
$$

Accordingly we split the quantities

$$
P=\left(\begin{array}{c}
P_{I} \\
P_{A}
\end{array}\right), \quad \Lambda=\left(\begin{array}{c}
\Lambda_{I} \\
\Lambda_{A}
\end{array}\right), \quad F_{q}=\left(\begin{array}{c}
F_{q I} \\
F_{q A}
\end{array}\right),
$$




$$
B=\left(\begin{array}{ll}
B_{\bullet} & B \bullet A
\end{array}\right), \quad D=\left(\begin{array}{cc}
D_{I} & 0 \\
0 & D_{A}
\end{array}\right) .
$$

Here and in the following $D$ is assumed to be diagonal. In the numerical experiments Raviart-Thomas elements of lowest order $R T_{0}$ (2d-example) or Taylor-Hood $P_{2}-P_{1}$ elements with a lumped $P_{1}$-mass matrix are used (1d- and $2 \mathrm{~d}$-examples).

Next we state the primal-dual active set strategy for solving (5.1).

\section{Algorithm.}

(1) Choose $c>0$. Initialize $P^{0}, \Lambda^{0}$. Set $k=0$.

(2) Set $A^{k}=\left\{i \in\left\{1, \ldots, n_{p}\right\} \mid \Lambda_{i}^{k}-c P_{i}^{k}>0\right\}, I^{k}=\left\{i \in\left\{1, \ldots, n_{p}\right\} \mid \Lambda_{i}^{k}-\right.$ $\left.c P_{i}^{k} \leq 0\right\}$.

(3) Solve for $\left(U^{k+1}, P_{I}^{k+1}\right)$ :

$$
\left(\begin{array}{cc}
M & B_{\bullet I} \\
B_{\bullet I}^{T} & 0
\end{array}\right)\left(\begin{array}{c}
U^{k+1} \\
P_{I}^{k+1}
\end{array}\right)=\left(\begin{array}{c}
F_{v} \\
F_{q I}
\end{array}\right)
$$

and set

$$
P_{A}^{k+1}=0, \quad \Lambda_{I}^{k+1}=0, \quad \Lambda_{A}^{k+1}=D_{A}^{-1}\left(F_{q A}-B_{\bullet A}^{T} U^{k+1}\right)
$$

(4) Stop, or set $k=k+1$, and return to 2 .

Hereafter we briefly comment this algorithm. For more details about the primaldual active set strategy with their local convergence properties we refer to [14] and the references therein.

- For the solution $(U, P, \Lambda)$ we have $P_{A}=0$ and $\Lambda_{I}=0$. However since the active and inactive sets depend on the solution itself, $A$ and $I$ are estimated during the iterations based on the current iterate.

- The above algorithm corresponds to a semi-smooth Newton method applied to the nonlinear system (5.1). In particular, the complementarity relation

$$
P \geq 0, \quad P \cdot \Lambda=0, \quad \Lambda \geq 0,
$$

is interpreted as

$$
\Theta(P, \Lambda)=\left(\Theta\left(P_{1}, \Lambda_{1}\right), \cdots, \Theta\left(P_{n_{p}}, \Lambda_{n_{p}}\right)\right)=0,
$$

where $\Theta(a, b)=a-\max (0, a-c b)$ for $a, b \in \mathbb{R}$ and a fixed $c>0$.

It is worth mentioning that a similar ideas has been used in $[15,19]$ with a standard finite element discretization. 
- Step 3. corresponds to the update step where a "linearization" of the maxterm is computed by using the generalized derivative

$\partial \max (0, P-c \Lambda)=\operatorname{diag}\left(d_{1}, \ldots, d_{n_{p}}\right) \quad$ with $d_{i}= \begin{cases}0 & \text { if } P_{i}-c \Lambda_{i} \leq 0, \\ 1 & \text { if } P_{i}-c \Lambda_{i}>0 .\end{cases}$

The involved linear system has a standard saddle point form. In our numerical tests, the built-in backslash Matlab solver gives excellent performances. However, for very fine meshes direct solvers becomes unfeasible and iterative methods have to be investigated. For more details about saddle point problem algorithms we refer to [7, 10].

- The value of parameter $c$ does not influence the convergence of the algorithm once we are sufficiently close to the solution. However $c$ should be tuned carefully if a globalization strategy is used. In the context of our time-dependant problem and for a reasonable time step size, the solution at a given time is expected to provide a good initial point for computing the solution at the next time step.

- As a stopping criterion we use $A^{k}=A^{k+1}$.

\section{NUMERICAL EXPERIMENTS}

In this section, we assess the practical performance of the proposed time-space discretization scheme and the solution algorithm described above. For this purpose, a Matlab code was written using the Getfem++ library [16]. Examples from the literature $[3,15]$ have been considered for validation and - for comparison purposes - publicly available source codes is used $[1,3]$.

6.1. Sinusoidal bearing profile. In our first example, we consider a steady-state one-dimensional problem with a hydrodynamic bearing of sinusoidal shape. The film thickness is defined as

$$
h(x)=h_{a v}-\Delta h \cos (2 \pi x / l), \quad x \in(-l / 2, l / 2)
$$

where $h_{a v}=0.02 \mathrm{~mm}, \Delta h=0.005 \mathrm{~mm}$ and $l=125 \mathrm{~mm}$. The sliding speed is $U=4 \mathrm{~m} / \mathrm{s}$, the lubricant viscosity is $\mu=0.015 \mathrm{~Pa}$.s and the cavitation pressure is $p_{c}=0 \mathrm{MPa}$. At the boundary the pressure is prescribed by $p( \pm l / 2)=1 \mathrm{MPa}$. The computation is stopped as soon as the difference in the maximum norm between two successive pressures is less than $10^{-12}$ with a time step $\tau=10^{-4}$. Figure 6.1 depicts the numerical solutions computed on uniform grids of size $d x=1 / 1000$. An excellent agreement has been found between the present formulation and the one proposed in [12]. 

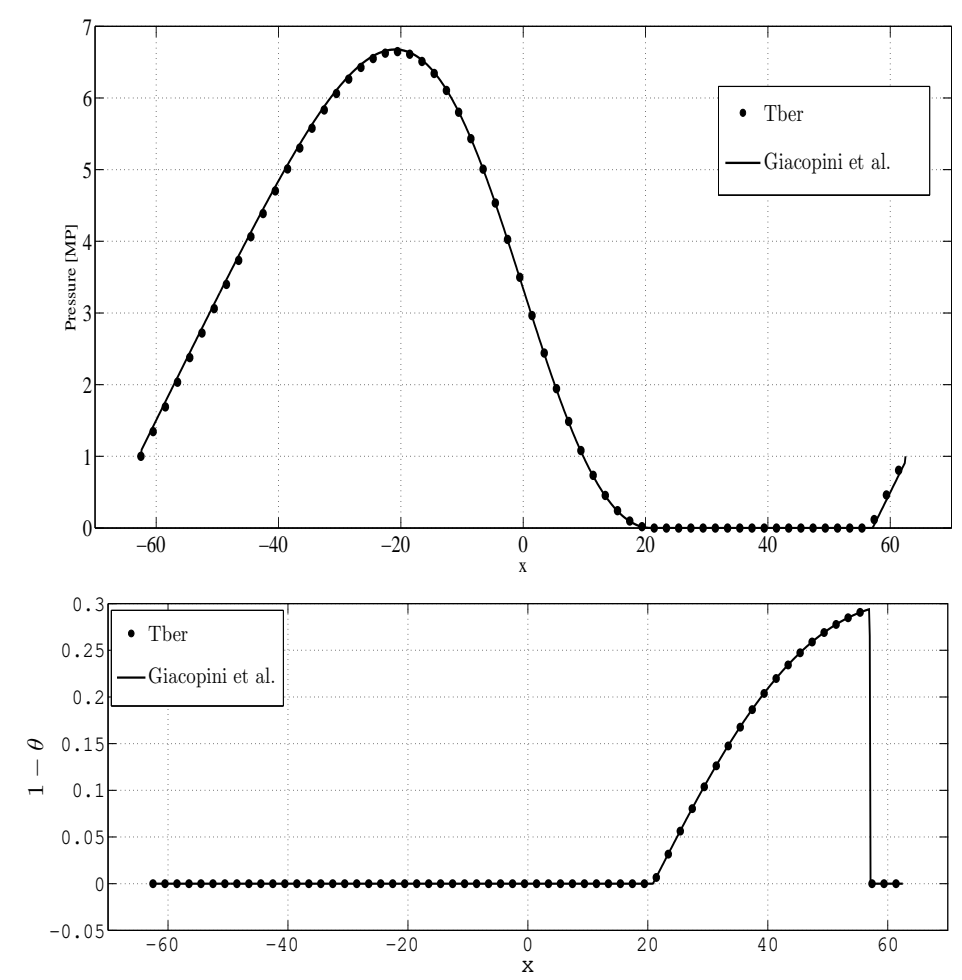

FIGURE 6.1. Solution of the 1D sinusoidal bearing problem: pressure (top) and void fraction (bottom)

6.2. Oscillatory squeeze flow. For the transient case we consider the classical example of two parallel plates in pure squeeze motion $(U=0)$ and separated by a lubricant with constant viscosity. The dimensionless formulation as presented in [3] is used with a computational domain $\Omega=(0,1)$ and a film thickness $h(x, t)=$ $H(t)=0.125 \cos (4 \pi t)+0.375$. On the boundary $p(0, t)=p(1, t)=0.025$. The initial condition is given by $\theta_{0}(x)=1$. For the numerical solution, a 450 -elements domain has been employed and the simulation time has been divided into 3000 steps. Figure 6.2 shows the variation in time of the extent of the cavitation zone. In comparison to Ausas et al. algorithm and the analytic solutions given in [3] a very good agreement was achieved. As mentioned there, the step character of the numerical result is due to discretization and could be made smoother by increasing the numbers of time steps and the points used for the discretization of the radius of cavitation area. In most stages of the simulation the active set solver converges in few iterations, though a large number of iterations is necessary during the transition from no-cavitation to with-cavitation phases. 

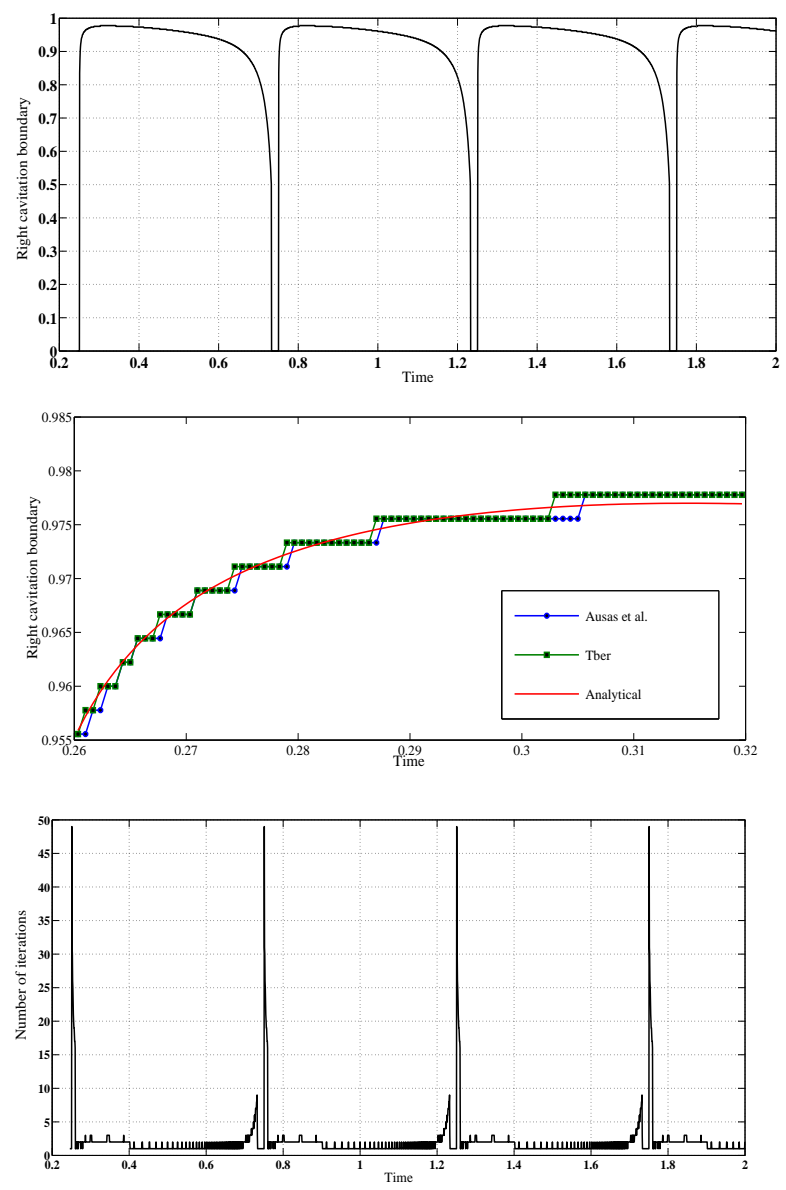

Figure 6.2. Pure squeeze problem: (top) time evolution of the right cavitation boundary (middle) comparison with Ausas et al. algorithm and the analytic method (bottom) number of the active set algorithm iterations with respect to time steps.

6.3. Sinusoidal bearing profile in $2 \mathrm{D}$. The sinusoidal bearing profile of the first example is extended here to two dimensions by considering

$$
h(x)=h_{a v}-\Delta h \cos \left(2 \pi x_{1} / l\right), \quad x \in(-l / 2, l / 2) \times(-l / 2, l / 2)
$$

and a constant pressure on the whole boundary $p=1 \mathrm{MPa}$. The steady state pressure and void fraction profiles obtained using $R T_{0}$ elements on a structured mesh with $100 \times 100$ elements are shown in figure 6.3. In the same figure, the number of iterations of the active set algorithm until successful termination is provided at each time step for different grids. Up to the first time step, the proposed algorithm converges in less than 10 iterations regardless of the mesh size. For the first time step a globalization strategy might be investigated to deal with the large number iterations. In fact, the standard result on the convergence of semi-smooth Newton methods requires an initial iteration sufficiently close to 
the solution. In figure 6.4 the computational results obtained using Taylor-Hood elements are shown. For this particular example better results are obtained. With $R T_{0}$ elements, the void fraction profile exhibits oscillations in the active-inactive sets boundary. Naturally with $P 2-P 1$ elements the cost in terms of computation time and memory is higher.

\section{REFERENCES}

[1] A. Almqvist, J. Fabricius, R. Larsson and P. Wall, A new approach for studying cavitation in lubrication, J. Tribol. 136(1), 11706 (2013). Code available at: http://www.mathworks.com/matlabcentral/fileexchange/41484

[2] T. Arbogast and M. F. Wheeler, A characteristics-mixed finite element method for advectiondominated transport problems, SIAM J. Numer. Anal. 32(2), 404-424 (1995).

[3] R. F. Ausas, M. Jai and G. C. Buscaglia, A mass-conserving algorithm for dynamical lubrication problems with cavitation, J. Tribol. 131(3), 031702 (2009). Code available at: http://www.lcad.icmc.usp.br/ buscaglia/codept08/index.html

[4] I. Babuska, The finite element method with Lagrangian multiplier, Numer. Math. 20, 179-192 (1973).

[5] G. Bayada, M. Chambat and C. Vázquez, Characteristics method for the formulation and computation of a free boundary cavitation problem, J. Comput. Appl. Math. 98(2), 191-212 (1998).

[6] G. Bayada and C. Vázquez, A survey on mathematical aspects of lubrication problems, Bol. Soc. Esp. Mat. Apl. 39, 31-74 (2007).

[7] M. Benzi, G. H. Golub and J. Liesen, Numerical solution of saddle point problems, Acta Numer. 14, 1-137 (2005).

[8] F. Brezzi, On the existence, uniqueness, and approximation of saddle point problems arizing from Lagrangian multipliers, RAIRO Anal. Numér. 8(2), 129-151 (1974).

[9] F. Brezzi, W.W. Hager and P. A. Raviart, Error estimates for the finite element solution of variational inequalities, Numer. Math 31(1), 1-16 (1978).

[10] H. C. Elman, D. J. Silvester and A.J. Wathen, Finite Elements and Fast Iterative Solvers with Applications in Incompressible Fluid Dynamics, in: Numerical Mathematics and Scientific Computation, (Oxford Univ. Press, New York, 2005).

[11] H. G. Elrod and M. L. Adams, A computer program for cavitation and starvation problems, in: Cavitation and Related Phenomena Lubrication (Mechanical Engineering Publications, New York, 1975) pp. 37-42.

[12] M. Giacopini, M. T. Fowell and D. Dini, A mass-conserving complementarity formulation to study lubrificant films in the presence of cavitation, J. Tribol. 132, 041702 (2010).

[13] D. Gropper, L. Wang and T. J. Harvey, Hydrodynamic lubrication of textured surfaces: A review of modeling techniques and key findings, Trib. Int. 94, 509-529 (2016).

[14] M. Hintermüller, K. Ito and K. Kunisch, The primal-dual active set strategy as a semi-smooth Newton method, SIAM J. Optim. 13(3), 865-888 (2003).

[15] J. Lengiewicz, M. Wichrowski and S. Stupkiewicz, Mixed formulation and finite element treatment of the mass-conserving cavitation model, Trib. Int. 72, 143-155 (2014).

[16] J. Pommier and Y. Renard, Getfem++: An open source generic C++ library for finite element methods, http://home.gna.org/getfem.

[17] P. Raviart and J. M. Thomas, A mixed finite element method for second order elliptic problems, in The Mathmatics Aspects of Finite Element methods, Lecture Notes on Mathematics, (Springer, Berlin), pp. 292-315. 

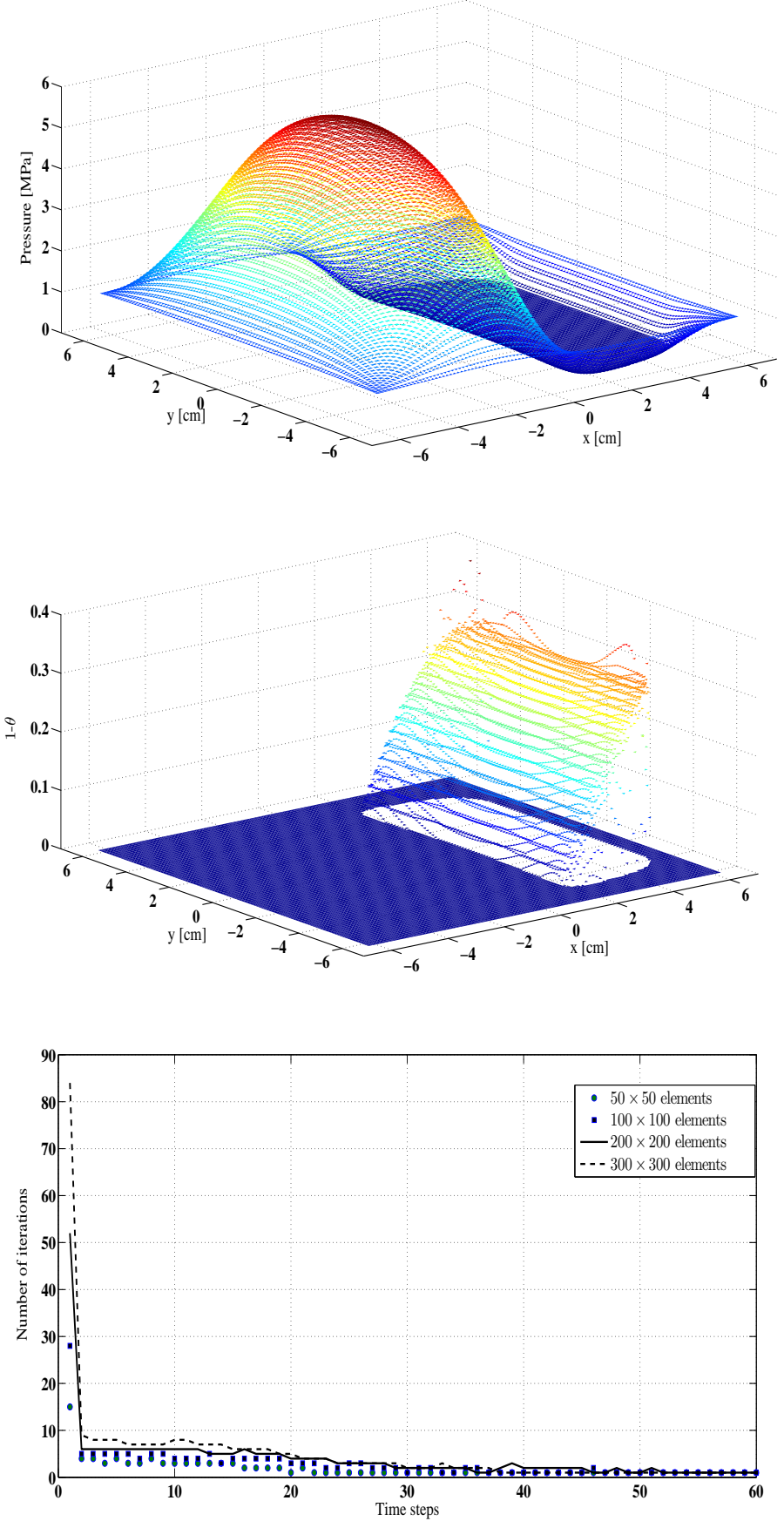

Figure 6.3. 2D sinusoidal profile: (top) pressure (middle) void fraction (bottom) number of the active set solver iterations with respect to time steps.

[18] L. San Andrés, Cavitation In Liquid Film Bearing, Lecture Notes on Modern Lubrication Theory, http://rotorlab.tamu.edu/tribgroup/default.htm.

[19] T. Woloszynski, P. Podsiadlo and G. W. Stachowiak, Efficient solution to the cavitation problem in hydrodynamic lubrication, tribol. lett. 58(1), 18 (2015). 

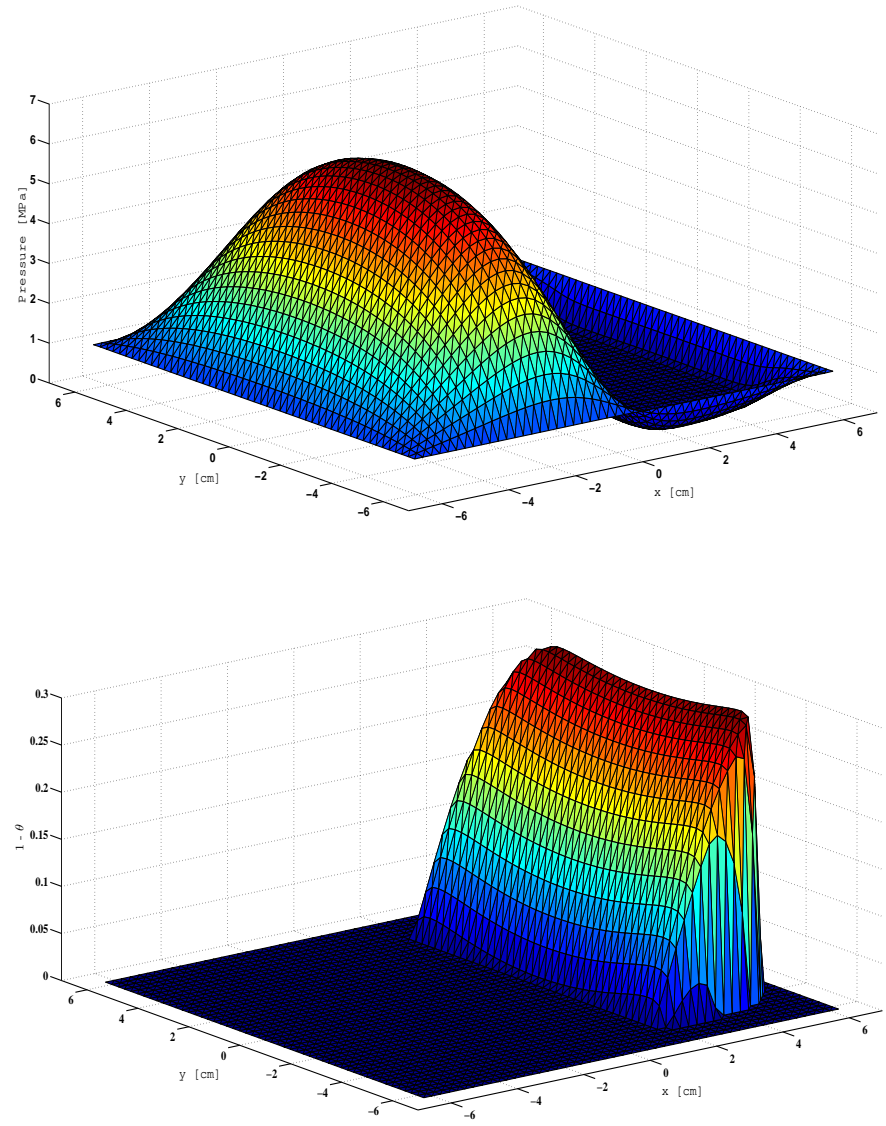

FiguRE 6.4. 2D sinusoidal profile with Taylor-Hoods elements: pressure (top) void fraction (bottom).

Cadi Ayyad University, F.S.T.G. B.P 549, Department of Mathematics, Av. Abdelkarim ElkhatTABI, MARRAKECH, MoRocco.

E-mail address: hicham.tber@uca .ac.ma 